\title{
Kernos
}

Revue internationale et pluridisciplinaire de religion grecque antique

7| 1994

Varia

\section{Sibylles, mantique inspirée et collections}

\section{oraculaires}

\section{Emilio Suárez de la Torre}

\section{OpenEdition}

\section{Journals}

Édition électronique

URL : http://journals.openedition.org/kernos/1107

DOI : 10.4000/kernos. 1107

ISSN : 2034-7871

Éditeur

Centre international d'étude de la religion grecque antique

Édition imprimée

Date de publication : 1 janvier 1994

ISSN : 0776-3824

Référence électronique

Emilio Suárez de la Torre, «Sibylles, mantique inspirée et collections oraculaires », Kernos [En ligne], 7 | 1994, mis en ligne le 20 avril 2011, consulté le 01 mai 2019. URL : http://journals.openedition.org/ kernos/1107 ; DOI : 10.4000/kernos.1107 
Kernos, 7 (1994), p. 179-205.

\section{SIBYLLES, MANTIQUE INSPIRÉE}

\section{ET COLLECTIONS ORACULATRES*}

\section{Introduction}

Peu de textes pourraient illustrer le sujet de ce Colloque mieux que la collection pseudépigraphe (d'origine juive, mais complétée et modifiée par des auteurs chrétiens) connue comme Oracles Sibyllins ${ }^{1}$. Cette affirmation est valable tant pour l'ensemble de la collection que pour le livre III, le plus ancien de tous, composé très probablement pendant le règne de Ptolémée VI Philométor ou, peut-être, de Ptolémée VIII

Cet exposé s'insère dans le cadre du Projet de Recherche PB 90/355, «Textos proféticos y oraculares en lengua griega", financé par la DGICYT du Ministère de l'Éducation Espagnol.

1. Après les grandes éditions commentées de Ch. AleXandre (Paris, I, 18411843; II, 1856; $1869^{2}$ ), nous disposons de celles de A. RzACH (Oracula Sibyllina, Wien 1891), J. GeFFCKen (Die Oracula Sibyllina, Leipzig 1902, réimpr. New York 1979) et A. KURFESS (avec traduction, Sibyllinische Weissagungen, Berlin 1951). Il est nécessaire de revoir profondément ces textes, ce qui a été entrepris partiellement par livres : le livre III a été étudié en détail et édité par V. NIKIPROWETZKY (La troisième Sibylle, Paris-La Haye 1970 - dorénavant cité "NIKIPROWETZKY"; voir aussi, du même auteur, Oracles Sibyllins, texte traduit, présenté et annoté par V. NIKIPROWETZKY, in La Bible. Ecrits intertestamentaires, Paris, Gallimard, 1987, p. 1035-1140 - seulement les fragments et les livres III et V); est récemment parue une bonne édition commentée du livre XIII : D. S. POTTER, Prophecy and History in the Crisis of the Roman Empire. A Historical Commentary on the Thirteenth Sibylline Oracle, Oxford, 1990. Du point de vue théorique, ce sont les livres III et V qui ont attiré le plus souvent l'intérêt des savants, l'étude récente la plus importante étant celle de J.J. CoLlins, The Sibylline Oracles of Egyptian Judaism, Missoula, Montana (The Society of Biblical Literature), 1974 (dorénavant cité "ColLINS"). Sur les problèmes posés par la collection en général, on peut consulter l'article Sibyllinische Orakel de A. RZACH, in $R E$, II A 2 (1923), c. 2103-2183, l'introduction de Kurfess, op. cit., celle de notre traduction commentée, Oráculos Sibilinos, in A. DiEz Macho (éd.), Apócrifos del antiguo Testamento, Madrid, Ediciones Cristiandad, 1982, vol. III, p. 241 sq. et la contribution de J.J. Collins, The Sibylline Oracles, in J.H. CHARLesWorth (éd.), Old Testament Pseudepigrapha, London, 1983, p. 317342. 
Évergète ${ }^{2}$. Ce texte est né en des circonstances historiques et sociales parfaitement connues qui expliquent bien les raisons de sa composition. La communauté juive d'Alexandrie avait vécu dans des conditions très différentes de celles de la communauté de Palestine, car la diaspora égyptienne n'avait pas connu de divisions internes (entre l'intégrisme et les philhellènes) et, surtout, la situation sous les Ptolémées était bien différente de celle vécue sous les Séleucides, qu'on pouvait qualifier pour le moins d'instable et toujours difficile ${ }^{3}$. Mais les choses vont changer radicalement pour les uns et pour les autres avec les débuts de la présence romaine dans ces territoires ${ }^{4}$. Les Séleucides ne connâ̂tront que des humiliations et des défaites. En ce qui concerne l'Égypte, après des bons débuts, la lutte dynastique a mis en évidence d'obscures intentions de la part de Rome. Ils ont appuyé d'abord Ptolémée VI Philométor, mais, en 161 av. J.-C., ils ont pris parti pour l'Évergète, ce qui, d'après la thèse de Collins ${ }^{5}$, aurait pu influencer l'opinion des Juifs, car le premier roi leur avait toujours été très favorable.

D'ailleurs, les événements des années suivantes vont renforcer toutes les craintes éveillées par la conduite des Romains. Un sérieux avertissement est donné en 146 lors des destructions brutales de Corinthe et de Carthage. En outre, la faiblesse des Ptolémées s'est accélérée immédiatement après. Dans le domaine culturel s'est produit aussi un fait extrêmement grave en 145/4: l'expulsion par Ptolémée VIII Évergète de tous les savants et érudits de la Bibliothèque d'Alexandrie. Peu après, il a dû lui-même abandonner définitivement la ville.

On peut comprendre que, dans ces circonstances, un Juif anonyme d'Alexandrie ait commencé à rédiger le noyau du livre III de cet

2 Des analyses plus détaillées des circonstances historiques qui entourent la naissance de cette collection se trouvent dans J.J. GEFFCKEN, Komposition und Entstehungszeit der Oracula Sibyllina, Leipzig, Hinrichs, 1902; NikIPROWETZKY, p. 195 sq. et ColLINS, p. 21 sq. et passim, ainsi que dans son article The Provenance and date of the Third Sibyl, in Bulletin of the Institute of Jewish Studies , 2 (1974), p. 1-18.

3 Bien qu'ils aient connu des moments de tranquillité (par exemple, sous le règne d'Antiochos III le Grand, 223-187 av. J.-C.), en général les Juifs de Palestine sont passés par des moments très difficiles, pour aboutir au désastre du royaume d'Antiochos IV, roi qui a eu l'honneur de devenir apocalyptique.

4 La bataille de Magnésie (189 av. J.-C.) signale l'irruption irrésistible des Romains en Asie Mineure et, surtout, dans le Moyen Orient.

5 Collins, p. 30. 
apocryphe vétéro-testamentaire. Au moment de le faire, il a entrepris un travail qui, tout en étant créatif dans une bonne mesure, peut être décrit comme synthétique et sélectif en même temps ${ }^{6}$. Cet auteur travaillait non sur une seule tradition, mais sur plusieurs, et non seulement juives ou grecques anciennes. En Égypte, par exemple, pendant les IIIe et IIe siècles av. J.-C. circulent des textes qui essaient de maintenir d'une façon un peu désespérée une conscience nationale et d'opposition aux dynasties helléniques, soit par l'exaltation de la royauté égyptienne soit par la prédiction de la ruine des nouveaux gouvernants, matérialisée dans la destruction de la ville d'Alexandrie. Les variétés littéraires employées dans ce but vont du genre narratif (Vie d'Alexandre) aux oracles (Oracle du potier), en passant par la chronique prophétique (Chronique démotique) ou le récit d'un songe, sans oublier le genre apocalyptique (Apocalypse du mouton de Bocchoris) ou les prophéties astrologiques (Nechepso-Petosiris) ${ }^{7}$. Le recours au genre prophétique est donc bien vérifié dans l'Égypte contemporaine de notre sibylliste en tant qu'expression d'une opposition, politique et religieuse, et d'affirmation nationale (ou, au moins, ethnique) en des moments critiques. On emploie surtout la langue de communication la plus efficace (en l'occurrence le grec, qui est aussi la langue de la classe dominante) et un langage prétendument traditionnel pour garantir «l'authenticité» de l'oracle.

Mais le sibylliste disposait aussi de traditions qui étaient sans doute plus anciennes et très complexes, dont l'analyse détaillée pourrait nous mener par des sentiers pleins de surprises. Cette analyse nous ferait aussi nous heurter à des problèmes un peu éloignés du monde grécoromain : qu'on pense à la polémique sur les antécédents orientaux du texte sibyllin tant du point de vue de la tradition littéraire (influence ou non d'une «Sibylle babylonienne» ${ }^{8}$ ) que de l'idéologie et des croyances

6 En ce qui concerne quelques traits importants de cette amalgame du point de vue de la tradition culturelle grecque, cf. J.M. NIETo IвÁÑEZ, Los Titanes y Noé : un ejemplo del sincretismo cultural de la comunidad judía de Alejandría, in $C F C$ (Estudios griegos e indoeuropeos), n.s. 1 (1991), p. 95-106; ID., El hexámetro de los oráculos sibilinos, Amsterdam, 1992.

7 Cf. un bon résumé de cette littérature et des précisions bibliographiques sur les éditions dans J.S. Fraser, Ptolemaic Alexandria, Oxford, 1972, I, p. 495 sq. avec les notes, II, p. 943 sq.

8 Cf. des positions diverses sur cette question dans A. Peretri, La Sibilla Babilonese nella propaganda ellenistica, Firenze, 1943, et NIKIPROWETZKY, p. 111 sq. 
qu'on y voit reflétées (parsisme vs. tradition juive "pure», etc. ${ }^{9}$ ), sans parler du propre prophétisme juif.

Dans cet article, je vais me borner à des questions concernant exclusivement la tradition grecque (mais sans éviter, et pour cause, le problème des origines «orientales» de la Sibylle). Mon but essentiel est d'établir qu'il faut différencier nettement deux aspects, malgré leur confluence dans la tradition sibylline: d'un côté, le problème de l'évolution de la mantique en Grèce et de ses figures (mythiques et historiques) les plus représentatives (en l'occurrence surtout de la Sibylle); et, de l'autre, le problème de la naissance et du développement des collections oraculaires en vers.

\section{Le problème des débuts de la mantique inspirée en Grèce}

Il serait trop hardi d'essayer d'être très original à propos de l'évolution de la mantique en Grèce. Il faut cependant dire deux mots du problème des origines orientales de la mantique inspirée en Grèce, inséparable de celui des origines supposées pour la Sibylle. Il est bien connu que le phénomène du prophétisme extatique, masculin ou féminin, est attesté dans les civilisations du Moyen Orient. Mais voyons comment on a cherché des solutions par cette voie. On a proposé, par exemple, que le parallèle lointain le plus étroit pourrait s'établir avec le royaume sémitique de Mari, dont les archives de l'époque de Hammourabi (1723-1689) contiennent (entre autres documents) des lettres destinées à Zimri-Lim avec des messages prophétiques des dieux (au nombre de neuf, le principal étant Dagan) ${ }^{10}$ communiqués par plusieurs intermédiaires. L'un d'eux est une femme appelée Shib-tou (qui est le nom de l'épouse de Zimri-Lim). On remarque ici une double difficulté. D'abord il faut employer les termes avec une grande prudence : mon opinion est qu'on ne peut parler que de précédents d'une mantique que nous trouverons développée (mais sous des formes très diverses) et dans le monde juif et dans le monde grec. Ensuite, les efforts qu'on a faits pour établir une parenté entre le nom de la Sibylle et

9 Ces composants ont été bien décelés par PeRETTI, op. cit. p. 363 sq.

10 Cf. C. Westermann, Die Mari Briefe und die Prophetie in Israel, in Forschung am Alten Testament, München, 1964, p. 171-188; F. ElLERMEIER, Prophetie in Mari und Israel, Herzberg, 1968, (textes p. 24 sq.; analyse des traits caractéristiques, p. 170 sq.); E. NooRT, Untersuchungen zum Gottesbescheid in Mari. Die "Mariprophetie" in der alttestamentlichen Forschung, Neukirchen, 1977. 
ceux des personnages féminins liés à la tradition prophétique orientale (Sambéthé, Shib-tou, etc. $)^{11}$ se heurtent à de graves objections, tant du point de vue chronologique que linguistique.

À de telles objections se heurtent d'autres propositions concernant non seulement la Sibylle, mais aussi la Pythie. Dowden ${ }^{12}$ est revenu sur le fait que la désignation des femmes qui transmettent le message des dieux est sha'ittu, "celle qui demande", tandis que le prophète-devin est appelé apilum, aplum et apillu, formes apparentées au verbe sumérien apalum, «répondre»; donc il est «celui qui répond». Cela permettrait, d'après l'auteur, d'expliquer d'un seul coup la fonction de la Pythie (en correspondance avec l'étymologie grecque) et la forme du nom du dieu Apollon. Il va de soi que je trouve des objections tout à fait pareilles à celles du cas précédent, auxquelles il faut ajouter les difficultés à admettre une antiquité de l'Apollon oraculaire delphique qui puisse s'adapter à cette explication ${ }^{13}$.

D'autres propositions ont pris en considération des civilisations non sémitiques, comme celle des Hittites, dont les documents épigraphiques nous offrent aussi des exemples de prophétie «extatique» ou, au moins, des prières faites par des prêtres "possédés" par le dieu (par exemple à l'époque de Mursil II, 1344-1315). En revenant à l'entourage sémitique, mais dans une période plus récente, nous avons des exemples importants à Ugarit et en Mésopotamie : c'est le cas des prêtresses du temple assyrien d'Ishtar à Arbela (pendant le royaume d'Asarhaddon, 680-669 av. J.-C.). Parke ${ }^{14}$ a également mis en relief l'intérêt des traits "sibyllins" de Balaam, qui apparaît en train de prophétiser devant les ambassadeurs de Balak dans la Bible (Num. 22-24) et dont on trouve le nom sur des inscriptions très importantes découvertes en 1967 dans le temple de Deir 'Alla. Dans ces textes Balaam, fils de Beor, est décrit comme un "voyant» qui communique avec Dieu à travers les rêves. Bien que cette inscription appartienne au VIII-VIIe siècle (ca. 700) et

11 Cf. W. BURKERT, Die orientalisierende Epoche in der griechischen Religion und Literatur, Heidelberg, 1984, p. 78-79.

$12 \mathrm{~K}$. DowDEN, Apollon et l'esprit dans la machine : origines, in REG, 92 (1979), p. 293-318 (vid. p. 309) et 93 (1980), p. 486-492.

13 Cf. nos remarques infra.

14 H.W. PARKE, Sibyls and Sibylline Prophecy in Classical Antiquity (edited by B.C. McGing), London/New York, 1988, p. 217 sq.; cf. les remarques des éditeurs de ces textes : J. HOFTIJZER - G. VAN DER KOOIJ, Aramaic Texts from Deir 'Alla, Leiden, 1976, p. 268 sq. 
qu'elle nous montre Balaam comme une figure d'un passé lointain, l'inscription sur pierre de ces oracles prouverait la valeur qu'on leur attribuait.

Ces exemples, ainsi que d'autres spéculations linguistiques dépourvues de rigueur, on contribué non seulement à renforcer l'idée d'une origine orientale de la mantique inspirée, mais aussi de la Sibylle, bien que personne n'ait pu expliquer jusqu'à présent ni la façon exacte ni le moment historique précis de cette adoption. Bien qu'il soit peut-être prudent de se contenter d'admettre l'existence d'une manifestation religieuse et culturelle issue de façon spontanée çà et là (sans écarter définitivement des "contacts» éventuels), il y a pourtant d'autres données qui peuvent être invoquées à l'appui de la thèse d'une influence orientale plus ou moins directe. Surtout, en des termes généraux, l'importance du phénomène de l'orientalisation pendant plusieurs étapes de la culture grecque est en tout point incontestable (il suffirait de penser à l'adoption de l'alphabet au VIIIe siècle, qui n'a sans doute pas été un fait isolé), ce qui a laissé des traces profondes dans la religion et dans le mythe. En ce qui concerne la divination, Burkert a établi parfaitement les modalités de ces emprunts (et surtout l'expansion des pratiques hépatoscopiques), favorisés par l'activité des devins et des mages "errants", enregistrés pendant l'Âge de Bronze et aussi en plusieurs étapes du Protogéométrique et du Géométrique ${ }^{15}$. Encore plus important est le rapport (aujourd'hui hors de doute) entre les cultes grecs d'Apollon et des cultes et des traditions orientales. Par des procédés différents (qui n'ont rien à voir avec les «jeux" étymologiques que nous avons critiqués ci-dessus), plusieurs savants sont arrivés à des conclusions semblables ${ }^{16}$ sur ce point, en ce qui concerne tant les traits et les fonctions du dieu que ses rituels. De tout cet ensemble on ne peut pas exclure le sanctuaire de Delphes, dont l'activité oraculaire ne semble pas avoir existé avant le VIIIe siècle av. J.-C. Par exemple, on a fait

15 W. BURKERT, op. cit. p. 43 sq.

16 Cf. W. BURKERT, Apollon und Apellai, in RhM, 118 (1975), p. 1-21; ID ., ResepFiguren, Apollon von Amyklai und die Erfindung des Opfers auf Cypern. Zur Religionsgeschichte der 'Dunklen Jahrhunderte', in GB, 4 (1975), p. 51-79; B.C. DIETRICH, The Dorian Hyacinthia, in Kadmos, 14 (1975), p. 133-142; ID., Some Evidence from Cyprus of Apolline Cult in the Bronze Age, in RhM, 121 (1978), p. 1-18; Ch. Auffarth, Der drohende Untergang. 'Schöpfung' in Mythos und Ritual im Alten Orient und in Griechenland, Berlin-New York, 1991, p. 130-131. 
remarquer à plusieurs reprises ${ }^{17}$ le rôle décisif de Delphes dans l'introduction de la périodicité ennaétérique de calcul d'années d'après le système babylonien, qui n'a pas été fixé avec exactitude en Babylonie avant 747 av. J.-C., pendant le royaume de Nabonassar, bien que ces cycles de huit ans aient été connus auparavant, indépendamment de l'adoption d'un calendrier systématisé ${ }^{18}$.

Malgré tous les efforts pour éclairer l'évolution du culte apollinien dans le sanctuaire delphique, la grande énigme reste sans solution : les origines de la Pythie et le commencement de la mantique inspirée. La polémique la plus récente peut être retracée jusqu'à la parution du livre de P. Amandry, La mantique apollinienne à Delphes. Essai sur le fonctionnement de l'Oracle, Paris 1950, surtout parce que cet ouvrage s'opposait à une tradition fortement enracinée (et pleine de topiques) sur le «délire» pythique ${ }^{19}$. Pourtant, aujourd'hui, la discussion est surtout centrée sur l'antiquité relative de la mantique inspirée en Grèce et, tout au plus, sur les modalités que celle-ci avait adoptées.

Par exemple, Dietrich a défendu l'antiquité de la mantique inspirée en Grèce, mais avec certaines précisions ${ }^{20}$. Les anciens cultes dans les grottes et les oracles chthoniens auraient été liés secondairement à la Sibylle. Les Nymphes (mises après en rapport généalogique avec la Sibylle) représenteraient cette ancienne croyance dans la prophétie extatique de type chthonien (sans laisser de côté le rapport avec les Muses et autres divinités "inspiratrices») et elles seraient la preuve d'une tradition qui pourrait remonter jusqu'à l'Âge de Bronze. Étant donné que la mantique inspirée est inconnue d'Homère et d'Hésiode, cette circonstance aurait permis la fausse déduction que l'Inspirationsmantik était une importation tardive.

17 M.P. NILSSON, Geschichte der Griechischen Religion, München, $1967^{2}$, vol. I, p. 646; B.C. DIETRICH, Reflections on the Origins of the Oracular Apollo, in BICS, 25 (1978), p. 2-18 (vid. p. 9).

18 On peut le déduire des légendes et des rites, crétois et delphiques, qui ont comme élément substantiel cette période et dont l'antiquité semble hors de doute.

19 Cf. les détails de cette polémique dans A. PIÑERo, Sobre la inspiración de la Pitia délfica. Breve historia de una polémica, in Durius, 3 (1975), p. 405-416 (vid. p. 410 pour quelques précédents des théories d'Amandry, qui n'ont pourtant pas connu un pareil écho). 
Cette hypothèse est très suggestive, mais le grand problème est l'impossibilité de suivre les traces des cultes qui pourraient confirmer la réalité de cette supposition si haut dans le temps. Certes, plusieurs données, très bien analysées par Dietrich ${ }^{21}$, permettent de supposer l'existence d'une tradition mantique (au moins "technique" ou inductive) très ancienne. Mais on ne peut pas aller plus loin. D'ailleurs l'existence, par exemple, d'un culte mantique chthonien à Delphes est maintenant plus difficile à soutenir, après la révision faite par Sourvinou-Inwood 22 . En tout cas on admet aujourd'hui que l'activité de l'Apollon oraculaire à Delphes ne peut pas remonter au-delà de la seconde moitié du VIIIe siècle av. J.-C. ${ }^{23}$ Pour compliquer un peu plus les choses, même les objets qui ont un lien avec le rituel mantique ne semblent pas à l'origine attachés à ces fonctions. C'est le cas du trépied qui est un instrument de sacrifice. Dès lors, le fait que la Pythie reçoive le dieu assise sur un tel instrument a amené Burkert à proposer que de cette façon la femme était offerte à Apollon comme une "restitution rituelle» après le sacrifice ${ }^{24}$. Il est vraiment difficile de décider si cette union si importante des composantes du rituel est une formule de compromis entre un élément plus ancien (trépied) et un autre plus récent (présence de la femme) ou bien on doit admettre une réinterprétation d'un rituel qui, à l'origine, n'avait aucune liaison avec la mantique. À mon avis, on peut compter sur une évolution dans la conception de la mantique qui, à un moment donné, a été mise en rapport avec un nouveau concept de possession divine pour lequel on ne peut ni refuser ni accepter définitivement l'origine orientale (et, quoi qu'il en soit, il faudrait écarter définitivement l'influence dionysiaque) ${ }^{25}$. Il faut compter également sur un rôle très important des traditions littéraires

21 Cf. B.C. DIETRICH, Oracles and Divine Inspiration, in Kernos, 3 (1990), p. 157 174.

22 Ch. Sourvinou-Inwood, Myth as History: The Previous Owners of the Delphic Oracle, in J. BREMMER (ed.), Interpretations of Greek Mythology, London, 1987, p. 215-241.

23 Cf. DIETRICH, Reflections... et l'interprétation des données archéologiques faite par C. Morgan, Athletes and Oracles. The Transformation of Olympia and Delphi in the Eigth Century B.C., Cambridge 1990, p. 106 sq.

24 W. Burkert, Homo Necans. The Anthropology of Ancient Greek Sacrificial Ritual and Myth (trad. angl. de Peter Bing de l'édition de Berlin 1972), Berkeley-Los Angeles-London, 1983, p. 235.

25 Cf. B.C. DieTrICH, Divine Madness and Conflict at Delphi, in Kernos, 5 (1992), p. 41-58. 
dans la création d'une sorte de mythologie sur le «délire» et, en général, sur tous les aspects du culte. Le plus important est que le culte apollinien, renouvelé dans cette orientation mantique, s'est enraciné profondément à Delphes, qui est devenue à partir du VIIIe siècle le centre de consultation par antonomasie.

S'il est un lieu où la mantique inspirée pourrait avoir laissé des traces, c'est, évidemment, la littérature, ce qui ne veut point dire que les poètes travaillent nécessairement au niveau du réel. Il n'y a pas encore d'opinion unanime des savants sur la présence de la mantique intuitive chez Homère; on ne peut citer qu'un seul passage à l'appui de ceux qui veulent la défendre. Certes, une analyse isolée d'Od., XX, $351 \mathrm{sq}$. pourrait faire croire que c'est un cas de prédiction produite par un état d'enthousiasme soudain. La description est vraiment tout à fait $s u i$ generis dans l'ensemble de la littérature archaïque. ${ }^{26}$ Rien dans la scène ne permet de penser à une déduction logique ${ }^{27}$ et il faut reconnaître que l'ensemble s'approche plutôt d'une vision apocalyptique. Pour Auffarth ${ }^{28}$, ceci serait le premier exemple de ce genre dans la littérature occidentale. Mais j'ai mis en garde contre une analyse isolée et il me faut démontrer ce que je veux dire.

Avant tout, il ne faut pas oublier que Théoclymène, depuis sa première apparition au chant XV (223-281), est présenté comme un mantis avec un "pedigree" sans tache : c'est un Mélampodide. Un mantis doit savoir ce qui se passe, ce qui s'est passé et, surtout, ce qui va se passer ${ }^{29}$. Il disposait d'un bon exemple dans sa famille: Mélampous avait prédit que, dans le délai d'une année, il reviendrait à Pylos avec les vaches de Phylaque pour son frère Biante. Rien d'extraordinaire donc en ce qui concerne la façon archaïque de concevoir les pouvoirs du

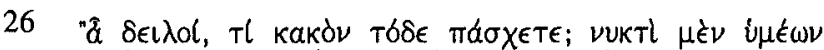

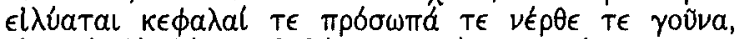

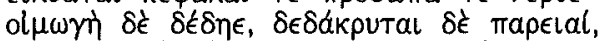

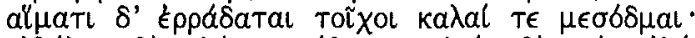

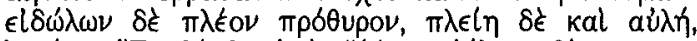

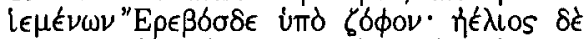

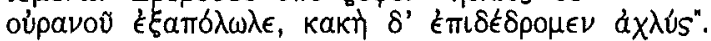

27 Contra DiETRICH, Oracles..., p. 161 : mais Théoclymène fait référence à ses yeux, ses oreilles, ses pieds et son noos d'une façon ironique, seulement pour indiquer qu'il n'a pas besoin de compagnie pour sortir de la salle (v. 364-370) et non comme explication de sa prédiction (cf. l'inclusion des "pieds»).

AUFFARTH, op. cit., p. 482-486.

Il., II, 70. 
devin $^{30}$. Ce qui peut nous surprendre c'est surtout la manière de décrire le «pressentiment» de Théoclymène, qui justifie la définition d'Auffarth. On peut admettre que ce passage, ainsi que d'autres détails, permettraient de dire avec Dodds que, dans le domaine de la divination, l'Iliade est plus riche en présages («omens»), tandis que dans l'Odyssée il y a "something more sensational» 31 . Mais cette tendance à l'expression apocalyptique au niveau descriptif ne doit pas nous induire en erreur au moment de chercher les traces de l'enthousiasme et de la possession divine. En plus, les éléments qui composent cette vision ne sont pas exclusivement «orientaux", mais (comme on l'a fait remarquer souvent) ce sont des «motifs» attestés dans d'autres littératures ${ }^{32}$.

Le personnage de Théoclymène ne peut pas être employé non plus pour attaquer le caractère unitaire de l'Odyssée. La cohérence du personnage est, à mon avis, hors de doute. Dans sa première rencontre avec Télémaque (et après le récit de sa généalogie de devins) il interprète un augure d'une façon favorable pour la famille d'Ulysse ${ }^{33}$ et en des termes généraux : il prédit la continuité de la dynastie locale, car ce genos n'a pas de rival. Au chant XVII, il fait une prédiction plus concrète à Pénélope : Ulysse est déjà en Ithaque et il prépare la fin des prétendants. À ce moment-là il explique quel était le sens concret de l'interprétation de l'augure qu'il avait donné à Télémaque ${ }^{34}$. Enfin, au chant XX, il «explose» devant les prétendants : le poète choisit pour ce moment de climax la description la plus terrifiante dont il pouvait disposer et, en même temps, il introduit des éléments qui anticipent les épisodes de la fin du poème tels que le sang, l'obscurité profonde des Enfers, les eidola des criminels qui vont errer à travers celle-ci, etc. ${ }^{35}$ En somme, un exemple parfait des aspects fonctionnels de la mantique dans la poésie archaïque grecque.

30 Cf. nos remarques dans Les pouvoirs des divins et les récits mythiques. L'exemple de Mélampous, in LEC, 60 (1992), p. 3-21.

31 E.R. DoDDs, The Greeks and the Irrational, Berkeley-Los Angeles-London, $1973^{8}$, p. 70, en parlant précisément de ce passage homérique.

32 Cf. DodDs, op. cit. p. 87. n. 38 et J. Russo in A Commentary on Homer's Odyssey, vol. III (Books XVII-XXIV), Oxford, 1992, p. 124.

$33 \mathrm{XV}, 531 \mathrm{sq}$.

34 XVII, 151 sq.

35 Cf. Russo, op. cit., ad loc. 
L'autre exemple littéraire qu'on emploie pour illustrer l'état de possession est Cassandre. Mais, pour cette figure, les conditionnements littéraires sont encore plus forts ${ }^{36}$, car c'est seulement le tableau désigné par Eschyle ${ }^{37}$ qui peut servir dans ce but. On dit que cette description de la transe faite par le tragique pourrait suivre le modèle «sibyllin» et, en général, serait un bon exemple (malgré le remaniement littéraire) de possession mantique féminine, même de la Pythie. Mais Graf ${ }^{38}$ nous a mis en garde contre cet "enthousiasme" chercheur. D'abord il fait remarquer que la Sibylle, dans les descriptions littéraires (tardives), ne tombe pas dans une extase sensu stricto. Normalement le "possédé» ne parle pas lui-même, mais il prête seulement sa voix au dieu (ainsi, dans les oracles transmis comme delphiques c'est normalement Apollon qui parle par la bouche de la Pythie), tandis que Cassandre et la Sibylle parlent elles-mêmes. D'ailleurs, les conditionnements du culte éloignent la Pythie du type Cassandre-Sibylle: la première est liée à la force conservatrice du rituel, tandis que les autres subissent un processus d'individualisation qui évolue selon les lois propres aux personnages mythiques. Ajoutons des différences chronologiques et géographiques. Cassandre n'a pas le don de prophétie chez Homère; on lui attribue des prédictions dans les Cypria ${ }^{39}$ (mais nous ne disposons pas de descriptions détaillées) et ce n'est qu'à partir des textes du Ve siècle qu'elle est décrite sous les effets de la possession (et, en même temps, en conflit avec Apollon) ${ }^{40}$. On

36 De même que pour Manto, où il y a aussi un intérêt à construire une «histoire» des rapports Delphes-Claros; cf. A. Bouché-LECLERCQ, Histoire de la divination dans l'Antiquité, Paris, 1880 (réimpr. Aalen, 1978), II, p. 150.

Ag, 1072 sq.

38 F. GRAF, Nordionische Kulte. Religionsgeschichtliche und epigraphische Untersuchungen zu den Kulten von Chios, Erythrai, Klazomenai und Phokaia, Rom, 1985, p. 347 sq.

Cyp. arg.; Il. exc. arg.; Il. parv. 15; Nost. 10 Bernabé.

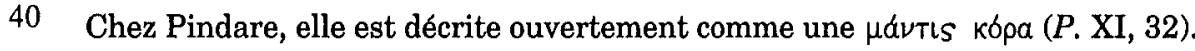
L'évolution de ce mythe fut étudiée par J. DAvREuX, La légende de la prophétesse Cassandre d'après les textes et les monuments, Liège-Paris, 1942. C'est en sortant du niveau littéraire qu'on trouve des données différentes et, apparemment, un peu inquiétantes. D'après la rigoureuse analyse linguistique faite par J. L. GARCfA-RAMÓN du nom de Cassandre (Mycénien ke-sa-do-ro /Kessandros/, ke-ti-ro /Kestilos/, ke-to /Kestor/: Grec alphabétique

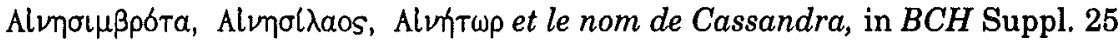
[1992], p. 239-255), attesté déjà sur les tablettes mycéniennes, au masculin et 
aperçoit aussi une confrontation entre le niveau mythique et la réalité du culte: Apollon veut posséder (même dans le sens sexuel) la prophétesse, mais celle-ci doit rester vierge pour être la prêtresse du dieu. La solution à ce conflit est diverse : Cassandre se refuse à Apollon et elle subit le châtiment de ne pas être crue dans ses prédictions, tandis que, dans les légendes sur la Sibylle, celle-ci renonce volontairement au dieu et, dans les Oracles Sibyllins, elle exprime à plusieurs reprises l'inutilité de sa résistance au dieu ${ }^{41}$.

\section{La Sibylle}

La Sibylle est sans doute le personnage représentatif de la mantique inspirée en Grèce dont les références dans les sources anciennes sont les plus abondantes. La difficulté reste toujours le caractère récent de la plupart de ces sources. Mais nous disposons de quelques indices pour attribuer une certaine antiquité à sa présence en Grèce ancienne et même à certains éléments des traditions qui la concernent. On commence d'habitude par la citation d'Héraclite, transmise par Plutarque ${ }^{42}$, qui nous fait une description assez remarquable de cette femme :

au féminin, avec le vocalisme radical -e - (ke-sa-do-ro, ke-sa-da-ra=

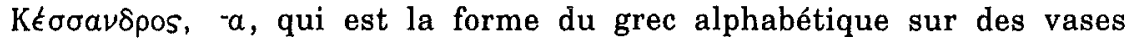
archaïques), la signification primitive de ce nom (dont le premier élément du composé appartiendrait à la racine IE *kens- = «faire des indications en ton emphatique») "s'accorderait bien avec le caractère de la prophétesse» (p. 248),

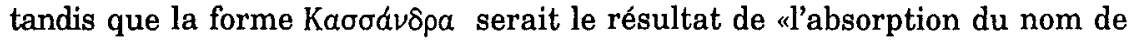

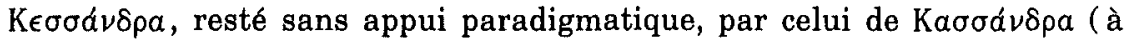
l'origine, "celle qui se distingue parmi les hommes")" (p. 255). On se heurte ici au fait paradoxal que les données linguistiques nous désignent un processus inverse de ce qu'on déduit des données littéraires. Mais il faut remarquer, d'abord, que la signification qu'on propose pour le radical IE est loin d'être «prophétique»; il s'agit d'un sens plus général, très en accord avec une fonction "masculine». Le féminin semble une dérivation mécanique du masculin, sans rapport déjà avec cette signification, dont la survivance d'ailleurs dans la conscience lingüistique même des mycéniens ne serait pas facile à expliquer. Il me semble plus prudent de renoncer à une spéculation sur une tradition mycénienne de la Cassandre prophétesse.

41 Or. Sib. , 2, 1-5; 346 sq.; 3, 1-7; 295-299; 489-491; 698-701; 11, 322-324; 12, 293$299 ; 13,172-173$; cf. la bibliographie sur les parallèles d'autres religions dans GRAF, op. cit., p. 347, n. 95.

PLUT., de Pyth. orac., 397a-b (= HÉRACL., fr. 92 DK, 75 Markovich). 


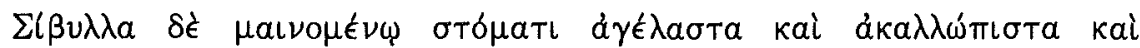

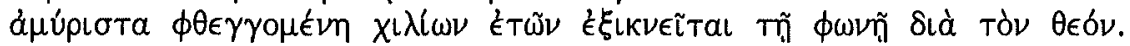

Le fragment n'a pas été moins discuté que celui qui décrit l'activité prophétique du dieu delphique. Les difficultés principales du passage sont d'ordre textuel (quelle est la partie qui appartient à Héraclite et qu'est-ce qui a été ajouté par Plutarque pour adapter la phrase au contexte) et d'ordre syntaxique, car les trois formes au neutre pluriel ne peuvent pas fonctionner comme "accusatif interne" (adverbialisé) du verbe $\phi \theta \epsilon \hat{\gamma \gamma o \mu a l}$ sauf comme emploi "métaphorique» dans un sens très large. Mais il faut remarquer que la Sibylle est citée dans une série d'exemples illustrant le pouvoir d'exercer un effet séduisant et charmant sur l'auditoire, malgré l'absence d'embellissement tant de l'interprète que du chant, en commençant par la Pythie ${ }^{43}$, laquelle ov

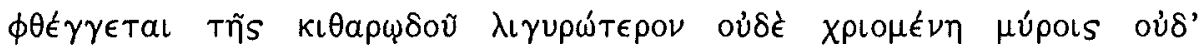

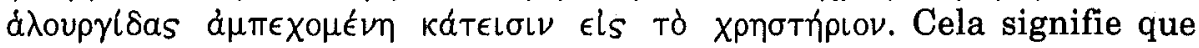

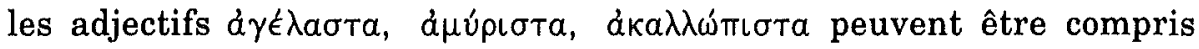
dans un sens littéral car, bien qu'à $\gamma \hat{\epsilon} \lambda a \sigma \tau a$ puisse être interprété en référence au contenu verbal («dire des choses qui ne font pas rire»), sa coordination avec les deux autres conduit à une interprétation purement adverbiale (modale): parler "sans rire, sans ornements, sans parfums». D'ailleurs on aperçoit des restes de formulation poétique en vers dactylique (cf. la séquence $\mu a \iota \nu o \mu \epsilon \nu \omega$ oTó $\mu a \tau \iota$ ou le participe $\phi \theta \epsilon \gamma \gamma \sigma \mu \epsilon\langle\eta)$ qui font penser à un remaniement ${ }^{44}$ d'éléments qui ont été, disons, maladroitement intégrés ${ }^{45}$. Retenons en tout cas, pour le moment, ces indices de diction hexamétrique, sur lesquels nous reviendrons.

En ce qui concerne le sens de la citation, Plutarque (on ne pourrait pas assurer que c'était l'intention d'Héraclite) veut souligner la force du message de la Sibylle, malgré la sobriété de sa "performance». Le seul argument pour admettre un état d'enthousiasme est le participe $\mu a \iota \nu o \mu \epsilon \nu \omega$, ce qui, d'ailleurs, n'est pas vraiment une «quantité

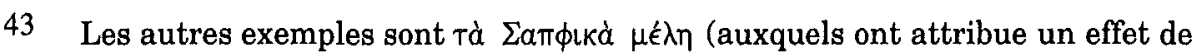

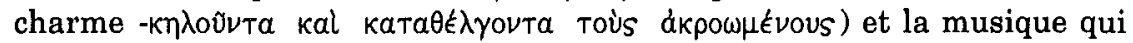

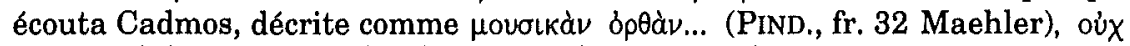

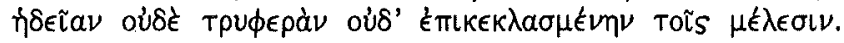

44 On ne peut pas écarter deux niveaux de citation : X-Héraclite-Plutarque.

45 Cf. les opinions de G. KIRK (in AFC, 83 [1959], p. 207) et M. MARCOVICH (Heraclitus. Greek text with a Short Commentary, Mérida, 1967, p. 403 ad loc.), qui n'admettent comme "genuine" que la séquence $\Sigma\left(\beta \nu \lambda \lambda \alpha \mu \alpha \iota \nu \mu \epsilon^{\prime} \nu \omega\right.$

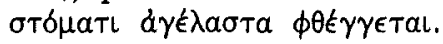


négligeable». Le seul problème serait de savoir quelle est la portée exacte du terme et il est impossible d'y répondre sans plus de données. En tout cas, on ne doit pas le rapprocher nécessairement, à l'appui de

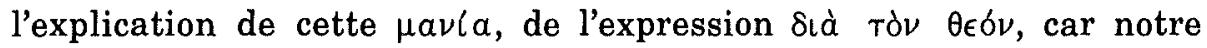
source semble mettre l'accent seulement sur la longue période de l'activité (ajoutons "prophétique») de la Sibylle, un phénomène qui s'est produit "à cause du dieu". Derrière ces phrases un peu énigmatiques pourrait se cacher une version ancienne du mythe que nous connaissons par Ovide ${ }^{46}$ : la Sibylle reçoit le don d'une extraordinaire longévité (précisément mille ans ${ }^{47}$ ), mais non celui de l'éternelle jeunesse, de sorte que, au fil des siècles, seule restera sa voix : uoce tamen noscar, uocem mihi fata relinquent ${ }^{48}$. Si l'ensemble provient d'Héraclite nous aurions ici le premier témoignage de ce thème ${ }^{49}$.

Après Héraclite (et dans une séquence purement chronologique), ce sont les auteurs du Ve siècle qui sont pris normalement en considération comme source pour connaître la Sibylle. La scène athénienne nous donne le témoignage de la popularité de ses oracles, surtout les comédies d'Aristophane ${ }^{50}$, où on remarque aussi une tendance à citer Bacis, la Sibylle et même la Pythie comme auteurs d'oracles "de collection". Quant aux autres genres dramatiques, Parke ${ }^{51}$ a traité de façon assez détaillée le problème posé par le drame satirique Bousiris. Lactance ${ }^{52}$ (qui cite Varron) et la Théosophie éditée par Erbse ${ }^{53}$ nous renseignent sur le prologue d'une pièce d'Euripide, qu'on croyait à tort intitulée

46 Mét., XIV, 101-153.

47 V. 144-146, nam iam mihi saecula septem acta uides : superest, numeros ut pulueris aequem, ter centum messes, ter centum musta uidere.

48 V. 153.

49 Hypothèse soutenue déjà par PARKE, op. cit. p. 20, n. 15 (et cf. ses remarques sur ce fragment d'Héraclite, p. 63).

50 Cf. N.D. SMITH, Diviners and Divination in Aristophanic Comedy, in CA, 9 (1989), p. 140-158, ainsi que notre contribution au IV Coloquio Internacional de Literatura Griega (tenu à l'U.N.E.D. de Madrid en mai 1993 et consacré à la Comédie), intitulée Observaciones sobre la presencia de la mántica en la Comedia Griega (à paraître dans les Actes).

51 Op. cit., p. 104-105.

52 Div. Inst. , I, 6, 8.

53 H. ERBSE, Fragmente griechischer Theosophie, Tübingen, 1941, p. 185, 1. 16. 
Lamie 54 , où l'on faisait mention (dans le prologue) de la Sibylle Libyenne. Nous savons aujourd'hui, grâce à un papyrus d'Oxyrhynchos étudié par Snell ${ }^{55}$, que cette œuvre était le Bousiris. Si Lamie parlait dans le prologue de la Sibylle, c'était sans doute parce qu'elle expliquait sa généalogie (et parlait de sa descendance). Les manuels de mythologie distinguent deux "Lamies": une femme à la mine épouvantable, qui habitait les caves et mangeait les enfants, et une fille de Poséidon, mère, par Zeus, de la Sibylle. La Lamie monstrueuse semble avoir des correspondances orientales: Burkert ${ }^{56}$ l'a mise en rapport avec la figure mésopotamienne de Lamme ou Lamasthu, une autre femme épouvantable qui avait aussi l'horrible habitude d'avaler des enfants.

En réalité, si on interprète littéralement les divers récits, il faudrait distinguer, en principe, trois Lamies. Mais je pense qu'il s'agit plutôt de développements secondaires d'un nombre plus réduit. L'historien Douris de Samos ${ }^{57}$ racontait la triste histoire d'une belle fille libyenne qui s'était unie à Zeus et qu'Héra avait punie par la mort de ses enfants. Folle de chagrin, elle poursuivait et tuait la descendance des autres femmes. L'imagination des auteurs avait ajouté plusieurs traits terrifiants : elle avait des yeux amovibles et, suivant une interprétation très littérale des "testicules sales" dont nous parle Aristophane ${ }^{58}$, on a proposé qu'il s'agissait même d'un être hermaphrodite ${ }^{59}$ : une sérieuse faute d'analyse du langage de la comédie, car cette allusion, comme l'a remarqué Sommerstein, décrivait Cléon, d'une seule volée, comme "assoiffé de sang, efféminé et au sexe sale» 60 .

Nous avons cité des sources qui parlent de sa localisation en Libye. Seul Nicandre ${ }^{61}$ raconte l'histoire d'une Lamie, appelée aussi Sibaris, qui habitait dans une cave au pied du Parnasse et qui terrifiait tous les paysans jusqu'à ce qu'Eurybate, amoureux d'une des victimes, la tue en

54 Fausse déduction de l'expression in Lamiae prologo.

55 POxy., 27, 2455, fr. 19.

56 BURKeRT, Die orientalisierende... p. 80-81.

57 FGrHist 56 F 38 Jacoby (= PLUT., Dem., 19).

58 Cf. Vesp., 1035 sq.; Pax, 758, etc.

59 Cf. J. BOARDMAN, art. Lamia, in LIMC, VI, 1 (1992), p. 189, avec une bonne bibliographie.

60 A. Sommerstein, The Comedies of Aristophanes, vol. 5, Peace, WarminsterChicago, 1985, p. 169.

61 Apud AnTON. LIB., 8. 
la frappant contre un rocher, d'où jaillit la source Sibaris. Néanmoins ce récit semble non seulement conditionné par l'aition, mais aussi établir un "compromis" avec la Sibylle delphique ${ }^{62}$. Plutarque ${ }^{63}$ nous dit que cette Sibylle était venue de l'Hélicon (où elle avait été nourrie par les Muses), mais que, d'après d'autres, elle était une fille de Poséidon et de Lamie venue du pays des Maliens, dont on sait par d'autres sources que la capitale s'appelait Lamie.

Mon opinion est que les versions delphiques (et, par conséquent, l'attribution d'une origine malienne) sont secondaires par rapport à la Lamie libyenne. D'un côté, on ne pouvait admettre l'ascendance "monstrueuse» de la Sibylle et, de l'autre, on voulait éviter la contradiction d'en faire la fille d'une femme qui avait perdu tous ses enfants.

En principe, quand Pausanias ${ }^{64}$ nous dit que, d'après les Grecs, Hérophile, dite Sibylle, était née de Zeus et de Lamie, fille de Poséidon, on ne pourrait pas affirmer qu'il s'agit de la monstrueuse Lamie ${ }^{65}$. Mais les notices sur le Bousiris d'Euripide nous permettent d'affirmer qu'au Ve siècle Lamie était pour les Athéniens une Libyenne et (compte tenu du genre dramatique) peut-être également un monstre. Il faudra donc admettre ces possibilités : soit le motif du châtiment d'Héra a été ajouté plus tard, soit il faut compter, effectivement, sur la fusion en un seul récit de deux Lamies, la mère de la Sibylle et une autre fille aimée de Zeus qui avait subi les effets de la colère d'Héra.

Parke a parfaitement expliqué le rapport entre le criminel qui tuait les étrangers sur l'autel (précisément !) de Zeus et Lamie : Bousiris est aussi un fils de Poséidon ${ }^{66}$, qui, de son côté, apparaît chez Hérodote ${ }^{67}$ comme un dieu des Libyens. En ce qui concerne l'argument du drame euripidéen, on pourrait supposer que le criminel recevait un oracle qui lui annonçait sa mort par les mains d'un étranger. Finalement, tout cet ensemble justifierait la présence de la Sibylle comme autorité oraculaire. Pour Parke il ne s'agit que d'une "pure fantaisie littéraire", sans fondement historique, et on peut en déduire que les Athéniens accep-

62 Pour la typologie de cette métamorphose, cf. P.M.C. Forbes IRving, Metamorphosis in Greek Myths, Oxford, 1990, p. 303-304.

63 Plut ., de Pyth orac., 398c; Souda, $\Sigma 355$ dit que le père était Apollon.

64 X, 12, 1 (et cf. PLUT., de Pyth. orac., 398c; schol. PLATON, Phèdre, 244b).

65 Cf. infra n. 74.

66 PHÉr., FGrHist 3 F 17 Jacoby.

67 HDT., IV, 88. 
taient sans problème toute localisation de la Sibylle ${ }^{68}$. Pourtant, nous disposons de quelques témoignages qui nous permettent de savoir dans quelle mesure tout est simplement une affaire de fantaisie littéraire d'Euripide.

Wilamowitz 69 se plaignait avec raison de l'impossibilité de connaître quels étaient les prédécesseurs de Thucydide qui, d'après Denys d'Halicarnasse ${ }^{70}$, avaient raconté des histoires sur les Lamies, qui habitaient des forêts, et les Naiades «amphibies" qui engendraient des demi-dieux. Je pense qu'aujourd'hui nous pouvons satisfaire Wilamowitz à titre posthume. En 1966, Barigazzi ${ }^{71}$ a attiré l'attention des philologues sur des passages de Favorinus qui contenaient des fragments des Corinthiaca d'Eumélos. Il s'agit des numéros 2 et 8 de l'édition de Bernabé ${ }^{72}$. Le fragment $n^{\circ} 8$ est cité à propos des traditions locales de Corinthe. La Sibylle y apparaît comme autorité pour confirmer la tradition de la célébration sur l'Isthme du premier agon sportif.

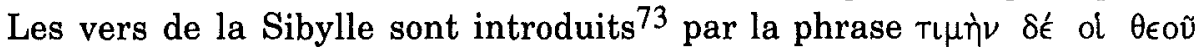

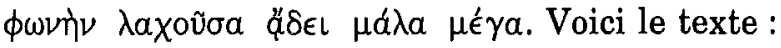

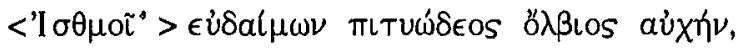

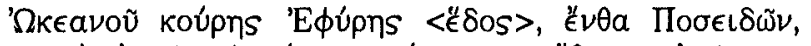

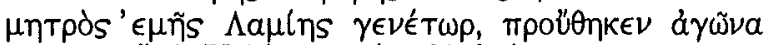

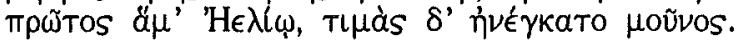

La Sibylle parle à la première personne, ce qui a sans doute provoqué une certaine méfiance dans l'attribution du fragment à Eumélos. Une prudence raisonnable amènerait à y mettre l'étiquette incerti auctoris, mais des problèmes assez importants subsisteraient, car il ne s'agit point, à proprement parler, d'un texte "sibyllin", mais d'une intervention à la première personne de la Sibylle dans un poème qui chantait l'histoire locale. Or, il n'est pas facile de trouver un autre poème local de

68 Op. cit., p. 105. À son avis le Bousiris pourrait être daté après le désastre de la Sicile, "when disillusionment with Oracle-mongers was at its height».

69 U. voN W Ilamowitz-MoEllendorf, Der Glaube der Hellenen, Darmstadt, $1955^{2}$ (réimpr. 1984) I, p. 267, n. 3.

70 Thuc., 6 (p. 333 Usener-Radermacher).

71 Nuovi frammenti dei Corinthiaca di Eumelo, in RFIC, 94 (1966), p. 129-148 (cf. Favorino di Arelate, opere, Firenze, 1966, p. 321-325).

72 Epicorum Graecorum Fragmenta, Leipzig, 1989.

73 FAvor. (= Dion CHRYs.), Corinth., 12 (305, 6 Barigazzi). C'est le fragment 12 Davies. 
cette nature en dehors de l'œuvre d'Eumélos et il est raisonnable de penser que Pausanias, qui connut un résumé en prose des Corinthiaca, employait cette source (ou bien une autre - delphique - influencé par la tradition «libyco-corinthienne»74) quand il exposait la même généalogie de la Sibylle. En tout cas, rappelons que Saint Jérôme établissait déjà un synchronisme entre Eumélos et la Sibylle d'Érythrées ${ }^{75}$.

L'importance de ce fragment est extraordinaire. La localisation corinthienne de Poséidon ne se heurte pas à des accusations «d'exotisme». L'intervention de la Sibylle était donc tout à fait naturelle, car le dieu était son grand-père. Le poète a par conséquent trouvé des éléments qui pouvaient être mis en rapport sans difficulté pour magnifier l'histoire locale... au risque que son œuvre fût qualifiée de $\mu \nu \theta \tilde{\delta} \delta \epsilon s$ par la postérité. Voilà donc la possibilité que je signalais : les Corinthiaca d'Eumélos qui prétendaient être une œuvre historique, mais qui étaient pleins de généalogies et de récits mythiques, peuvent être l'une des œuvres sans nom critiquées par Denys d'Halicarnasse. Et il faudra y ajouter d'autres récits locaux éventuels qui avaient profité du nom de la Sibylle pour garantir la "vérité» de leur contenu ${ }^{76}$.

Les conséquences sont aussi très remarquables en ce qui concerne la chronologie. Il faut rappeler d'abord que celle d'Eumélos pose de sérieux problèmes. Pausanias affirme que la seule œuvre authentique était le prosodion dont nous sont parvenus deux vers ${ }^{77}$, décrits par Pavese comme le "premier texte lyrique» en Grèce. La chronologie de ce fragment est difficile à établir, mais les hypothèses les plus convaincantes le placent à la fin du VIIIe siècle. Même en acceptant une datation plus récente, on n'arrive pas en deça du VIIe siècle. Quant aux fragments épiques attribués à Eumélos, on conserve des doutes sur leur authenticité et il pourrait s'agir d'un phénomène de pseudépigraphie : la

74 N'oublions pas que PAUSANIAS (X, 12, 1 sq.) mentionne cette généalogie dans l'excursus delphique sur la Sibylle et il parle seulement des «Libyens». PARKE, op. cit., p. 39, est de l'opinion que cette Lamie n'est pas la femme monstrueuse, mais la reine éponyme de la capitale des Maliens (cf. Plut., Pyth. orac., 9). Dans ce cas là il faudrait admettre que Pausanias veut dire que c'est seulement le nom qui est dû aux Libyens.

75 JÉRÔME, II, 83 Schöne : Eumelus corinthius versificator agnoscitur (T8 Bernabe) et Sibylla Erythraea.

76 Cf. infra les hypothèses de Parke.

77 C.O. PAVEsE, Il più antico frammento di lirica corale greca, in Studi Della Corte, Urbino, 1987, I, p. 53-57. 
plupart de ces textes auraient été composés pendant le VIe siècle sous le nom du grand poète local. Quoi qu'il en soit, cela veut dire que nous avons gagné au moins presque un siècle dans la recherche des témoignages grecs sur la Sibylle.

Revenons sur le problème de Lamie et de sa conjonction "généalogique» avec la Sibylle. Je pense que ce rapport a vu son origine (ou s'est consolidé) dans la tradition corinthienne que nous venons de commenter. Et je ne trouve pas d'autre justification que l'effort des poètes corinthiens pour établir un «tout» cohérent dans le cadre qu'il s'efforçaient de construire. Nous avons vu d'abord, à propos du Bousiris d'Euripide, quelques motifs de la relation entre la Sibylle et Corinthe. Il y a également d'autres raisons complémentaires. Je pense à l'importance des traditions locales sur Médée et, par conséquent, au mythe des Argonautes ${ }^{78}$. Dans ce mythe, la Libye joue un rôle naturel qui a pu être employé par les poètes pour introduire le personnage de la Sibylle. Rappelons aussi quelques traits de Médée (connus de la tradition locale) qui sont très importants pour nos déductions. Elle n'est pas seulement une magicienne, mais possède aussi des pouvoirs prophétiques (cf. Pindare). Elle a refusé l'union avec Zeus pour éviter la colère de Héra (dans ce sens elle est aussi ípo $\phi(\lambda \eta)$ et, par conséquent, elle a reçu la protection de la déesse. Voilà donc comment le travail des poètes nous laisse entrevoir un vrai «système», un constructum parfaitement harmonieux qui établit un lien entre les cultes et les traditions mythiques: Hélios-Médée en regard de Poséidon-Sibylle. Une Sibylle qui, dans son nom, porte aussi mystérieusement l'indication de son origine : $\Sigma(\beta v \lambda \lambda a \Lambda(\beta v \sigma \sigma a$, un jeu de mots déjà remarqué par BouchéLeclercq ${ }^{79}$, mais contredit par Rzach ${ }^{80}$. Faut-il rappeler que pour Pausanias ce sont les Libyens qui les premiers ont donné à cette femmeprophétesse le nom de Sibylle ${ }^{81}$ ?

J'ai présenté jusqu'à maintenant les témoignages d'auteurs qui pouvaient nous aider à arriver à une limite chronologique ancienne seulement parce qu'ils appartenaient à cette période. Il y a d'autres

78 Sur l'importance de cette légende à Corinthe et son rôle dans les Corinthiaca d'Eumélos, vid. B ARIGAzZI, art. cit. p. 140 sq.

Op. cit. , vol. 2, p. 161.

$80 \quad R E 2 \mathrm{~A} 2075$.

$81 \mathrm{X}, 12,1$. 
témoignages qui ont été employés pour arriver à une datation encore plus ancienne pour l'existence d'une tradition sur la Sibylle. Parke est arrivé jusqu'au VIIe siècle av. J.-C. à partir des renseignements que nous trouvons chez Pausanias, dans un fragment d'Ératosthène et dans un autre de Nicolas de Damas. Le périégète ${ }^{82}$, après avoir cité un oracle de la Sibylle et un autre de Musée concernant la bataille d'Aigos Potamos, ajoute que la première avait aussi prédit que dans la lutte entre les Lacédémoniens et les Argiens pour la possession de la Thyréatide les deux parties finiraient à égalité. Nous savons que cette confrontation a déjà commencé au VIIe siècle et s'est prolongée durant le VIe. On ne peut savoir à coup sûr si cette tradition «sibylline» est née immédiatement après les événements ou si elle a été forgée plus tard. À mon avis, le synchronisme établi par Hérodote ${ }^{83}$ entre cette guerre et la lutte de Crésus contre Cyrus, ne peut trancher la question, malgré la théorie suggestive émise par Parke : le récit d'Hérodote dénoncerait une tradition «apologétique» de l'absence des alliés spartiates pendant le siège de Sarde, à laquelle se serait ajouté très tôt la description sibylline des événements ${ }^{84}$.

Passons à Ératosthène ${ }^{85}$. D'après Varron, le grand érudit avait trouvé des données sur la Sibylle de Samos dans des anciennes annales gardées par les Samiens ${ }^{86}$. On suppose ${ }^{87}$ que la datation d'Ératosthène (qui avait écrit une Chronographia) était proche de celle que nous trouvons chez Varron (notre source), qui place la Sibylle samienne après celle d'Érythrées, et en rapport aussi avec la chronologie d'Eusèbe

\section{$82 \mathrm{X}, 9,12$ \\ $83 \mathrm{I}, 82,3$.}

84 PARKE, op. cit. p. 101 sq. Récemment N. ROBERTSON (Festivals and Legends. The Formation of Greek Cities in the Light of Public Ritual, Toronto-BuffaloLondon, 1993) a analysé la version d'Hérodote par rapport à celles de Plutarque et de Pausanias et il a défendu aussi le caractère artificiel (avec une tendance propagandiste) de la tradition sur les héros de la «bataille de Thyréa" : il s'agirait d'une légende créée à partir de la tradition née autour de la tombe d'un héros primitif, Otriadès, laquelle est devenue dans les récits le "tumulus" des champions tombés (p. 179 sq.). En réalité, d'après cet auteur, Sparte aurait conquis la Thyréatide au commencement du Ve siècle et non au temps de Théopompe ou de Crésus (p. 191-194).

FGrHist 241 F 26 Jacoby.

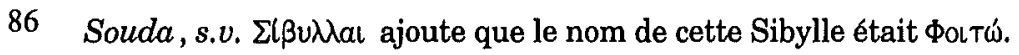


et de Saint Jérôme ${ }^{88}$, qui nous donnent les dates de 712-11 et 666-5. Bref, il faudrait la placer en tout cas avant 550 av. J.-C.

Quant à Nicolas de Damas ${ }^{89}$, il nous transmet un récit sur la mort de Crésus dans laquelle intervient la Sibylle. La déduction de Parke ${ }^{90}$ est qu'il ne s'agit pas d'une invention de l'auteur, mais que cette version provient de l'historien Xanthos de Lydie. C'est-à-dire qu'il existait une tradition sur des prédictions de la Sibylle concernant la fin des Mermnades.

Si les dernières remarques restent dans le domaine des hypothèses, bien qu'elles ne soient pas privées de probabilité, d'autres traditions sur l'antiquité de certaines "Sibylles" nous posent des problèmes assez délicats. Plusieurs sources visent à accorder une remarquable antiquité aux Sibylles de la Troade. Mais les récits et les traditions qui nous en parlent sont sans doute des élaborations tardives qui cherchent à démontrer cette antiquité d'une façon parfois trop artificielle. C'est, par exemple, le problème de la fameuse Sibylle d'Érythrées, dont les sources les plus anciennes appartiennent au IVe siècle : Héraclide du Pont et Callisthène, l'historien d'Alexandre ${ }^{91}$. Un historien local, Apollodore, nous dit qu'elle avait prédit la guerre de Troie ${ }^{92}$. Il va de soi qu'il s'agit ici d'un effort "patriotique» ad maiorem gloriam Sibyllae, qui a eu un grand succès. En fait, à Érythrées, nous avons l'un des rares sanctuaires sibyllins dont l'étude a pu être réalisée, bien que les restes trouvés en 1891 aient presque disparu. Les descriptions des archéologues qui ont fait les premières fouilles, ainsi que les inscriptions, nous permettent de nous faire une certaine idée des choses. Or, en vérité, comme le fait remarquer Graf ${ }^{93}$, ce qu'on obtient n'est pas une connaissance de la réalité du culte, mais plutôt de la façon dont les habitants ont conçu l'activité de la prophétesse et de la mythification locale de celle-ci. Par contre, c'est aussi Graf qui a prévenu contre la tendance à considérer comme trop récent le début de l'activité dans le sanctuaire, seulement par le fait que les sources sont tardives. Il suggère que la

88 Cf. le commentaire de F. JACOBY, FGrHist II B, p. 713.

89 FGrHist 96 F 68 Jacoby.

90 PARKE, op. cit., p. 60-61.

91 Héracl. PonT., fr. 130 W.; Callisth., FGrHist 124 F 14 Jacoby.

92 FGrHist 422 F 1 Jacoby; cf. PAUS., X, 12, 2 (ici la tradition est mêlée à une version délienne).

Op. cit., p. 337 sq. 
célébrité de celui d'Érythrées a commencé après avoir ratifié la nature divine d'Alexandre lors de sa visite à l'oasis de Siwa en 331 av. J.-C., épisode transmis par Callisthène ${ }^{94}$. Cet événement aurait provoqué la rivalité entre d'autres sanctuaires (peut-être plus anciens) et celui d'Érythrées, d'où l'origine de la controverse sur le lieu de naissance de la Sibylle.

Pausanias commente la rivalité entre Érythrées et le petit village de Marpessos, dont les habitants soutenaient qu'elle était la patrie de la Sibylle. Pausanias était d'accord, car son territoire correspondait à la description faite par la Sibylle dans la collection oraculaire qu'il possédait. Le terrain et le fleuve présentaient le ton rougeâtre décrit dans un fragment des vers de cette Sibylle qu'il nous transmet:

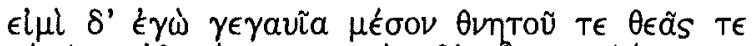

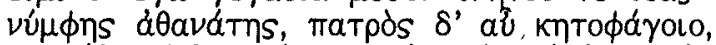

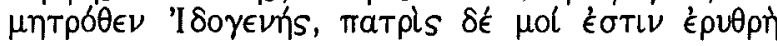

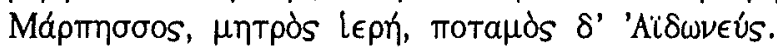

Les Érythréens soutenaient que le dernier vers était une addition des Marpessiens et les opinions les plus récentes leur donnent raison, surtout parce que le toponyme d'Érythrées est attesté aussi pour Marpessos et parce que Marpessos au temps de Pausanias n'était qu'un tout petit village de 60 habitants : la tradition, on le déduit, doit être plus ancienne. En outre, Marpessos n'a fait qu'hériter de la tradition de Gergis, ville disparue déjà au IIe siècle ${ }^{95}$, ce qui nous donne un terminus ante quem. Graf remarque avec raison que le terminus post quem doit être l'activité des historiens du IVe siècle qui se sont occupés de la tradition sibylline. En tout cas, la source de Pausanias doit être un historien local de la période hellénistique postérieur à Héraclide, car celui-ci place la Sibylle de Marpessos à l'époque de Cyrus et de Solon.

\section{Sibylle, Sibylles et collections oraculaires}

Le choix que je viens de faire des données les plus remarquables qui peuvent être employées pour établir une chronologie de la présence la plus ancienne de la Sibylle en Grèce exige de nous une bonne dose de prudence. L'itinéraire qu'on peut en déduire peut être très différent suivant la valeur que l'on attribue aux sources et aux traditions. La voie

\footnotetext{
94 FGrHist 124 F 14 Jacoby (cf. n. 90).

95 Cf. GRAF, op. cit., p. 341.
} 
proposée par Parke peut se résumer de la façon suivante : d'un premier centre "sibyllin" en Troade (Marpessos, possible héritière de Gergis), cette tradition serait passée aux territoires ioniens (Érythrées) et ensuite à Samos au cours du VIe siècle. À leur tour les Samiens auraient emporté la tradition sibylline en Italie (Cumes). Pour Parke il ne s'agit pas seulement du «culte» sibyllin, mais aussi de la composition des oracles. On se heurte ici à deux objections. D'abord, la tradition sur les sanctuaires sibyllins manque, pour la plupart des cas, de confirmation archéologique. D'ailleurs, il est très probable que la tradition littéraire a donné naissance plus tard à la tradition de l'existence d'un sanctuaire. En deuxième lieu, il faut distinguer radicalement les données sur la ou les Sibylles des prédictions qui leur sont attribuées, qui doivent être étudiées sans les mêler à la question de la Sibylle et de ses sanctuaires. Collins ${ }^{96}$ a fait remarquer qu'aucun des oracles transmis comme sibyllins n'appartient à un sanctuaire déterminé et que les références en Grèce ancienne à ces oracles sont beaucoup plus vagues que celles que nous possédons sur les collections d'autres auteurs non moins irréels (Orphée, Musée, Bacis).

Nous avons insisté sur quelques indices de tradition versifiée ancienne : rappelons nos remarques sur le témoignage d'Héraclite et sur le fragment attribué à Eumélos. Le cas d'Onomacrite nous sert de comparaison pour l'existence de collections d'oracles susceptibles de manipulations au VIe siècle. Au Ve siècle, il n'y a pas de doute sur l'existence d'un style et d'une structure oraculaire «sibylline» consolidée (peu importe si l'auteur reconnu le plus souvent est Bacis). Hérodote et Aristophane montrent qu'à côté de ces traits formels, il y a aussi une tendance à réserver pour ces oracles deux thèmes : la destruction des villes ou des pays et la succession au pouvoir. On s'aperçoit que ces oracles sont nés dans un but politique : le développement ultérieur va consolider cette tendance à les employer comme instrument de protestation, de propagande ou, tout simplement, pour créer un état d'opinion.

La Sibylle grecque a donc des origines très différentes de celles de la Pythie ${ }^{97}$. Il s'agit d'une invention littéraire, issue d'une période de

Coluins, p. 1.

97 Le témoignage de Platon (Phèdre, 244a-b), qui semble traiter pareillement la Sibylle et la Pythie, n'est pas un obstacle à cette hypothèse : une lecture détaillée du passage montre qu'il mentionne d'abord les prêtresses de Dodone et la Pythie en tant que représentatives des institutions prophétiques et qui ont fait des bénéfices à la Grèce avec sa «folie» mantique, tandis qu'il établit après

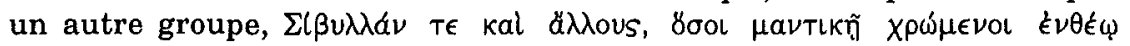


convulsion politique, et créée par des poètes (peut-être un poète) de la Grèce orientale, avec tous les éléments garantissant un grand succès. D'abord, il fallait compter sur la vraisemblance de la figure de la "femme inspirée», ce qui ne posait pas des problèmes dans le cadre général de la tradition grecque et encore moins pour les voisins des cultures plus proches du point de départ hypothétique de cette création. Car, comme l'a souligné Graf, "on ne peut pas concevoir cette création sans le tréfonds de la prophétie extatique réelle, telle qu'on peut la conjecturer pour l'Asie du Nord-Ouest” 98 . Puis, il faut y ajouter : le rapprochement de la mantique non apollinienne (Nymphes, grottes, des traits dionysiaques parfois, etc.) et également de l'ascendance olympienne; l'absence de rattachement à un lieu (c'est-à-dire, la version féminine des "wandering seers"), ce qui permettait la diffusion de la figure et des prophéties dans d'autres régions; la consolidation (en partant de la langue épique) d'un formulaire et d'une structure de la prédiction plus ou moins "typique». Quant aux thèmes, une nouveauté qui pourrait dénoncer l'influence "orientale": la récurrence du schéma de succession, non dans sa version "universelle", mais limité à une échelle plus réduite, et de l'annonce de dangers et de catastrophes imminentes.

Les conséquences de cette “invention" (adaptation?) vont être enregistrées dans plusieurs directions. L'extension géographique étant très facile (on pouvait altérer le prooimion du poème avec la généalogie et les indications du lieu d'émission du texte), la «Sibylle» a commencé à apparaître en rapport avec des lieux concrets. Mais cela ne veut pas dire qu'il s'agit en principe de l'existence de sanctuaires sibyllins, un

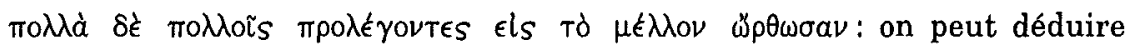
facilement que ces «autres» sont Orphée, Musée, Bacis et que Platon met ensemble les auteurs de prédictions qui n'appartiennent pas à un sanctuaire (donc «de collection» ou «littéraires»). Quoi qu'il en soit, même en admettant qu'il parle d'une Sibylle concrète (par exemple, celle de Delphes), son témoignage est déjà un peu tardif et le développement "cultuel" pourrait avoir déjà commencé en Grèce. Sur les problèmes posés par la Sibylle delphique (qui ne doit pas être considérée nécessairement comme tardive), cf. GraF, op. cit., p. 340, n. 44.

98 Op. cit., p. 349. Il ajoute : "Wie im Einzelnen der Sprung von der lokalen Ekstatikerin zum panhellenisch bekannten hexametrischen Spruch der Sibylle sich vollzog, ob eine bestimmte Prophetin besonders hervortrat, muss und kann auch, da nichts auf eine besondere Rolle Erythrais in diesem Prozess hinweist, unerörtet bleiben». Je pense qu'on ne peut pas effectivement affirmer qu'il y ait eu en Grèce une "lokale Ekstatikerin» à l'origine de la tradition. 
phénomène qui, à mon avis, appartient à une phase secondaire, bien qu'on puisse admettre que les poètes ont incorporé très tôt des indications plus ou moins précises et des descriptions de lieux qui ont été «identifiés» (rochers, grottes) a posteriori. Je me demande même à quel point est il raisonnable de s'efforcer à identifier $q u i$ était la Sibylle d'Héraclite et surtout quel était ce sanctuaire sibyllin ${ }^{99}$. D'autre part, si la Sibylle annonçait les événements les plus décisifs de l'histoire humaine, on ne pouvait pas en exclure (du point de vue des Grecs) la guerre de Troie, un motif qui peut appartenir aux couches les plus anciennes de la tradition, mais qui peut aussi avoir été ajouté plus tard, surtout en rapport avec la dispute pour la possession du sanctuaire le plus ancien de la Sibylle ${ }^{100}$.

On ne peut pas dire si la naissance du phénomène sibyllin en Grèce est due à un intérêt pour faire concurrence aux sanctuaires apolliniens ni les raisons qui auraient pu contribuer à ce phénomène, bien qu'on puisse établir une série d'hypothèses à sujet. En tout cas, le plus souvent, la Sibylle a été "soumise», dans tous les sens, à Apollon et on est arrivé à une parfaite compatibilité partout. Le grand avantage de la prophétie sibylline est qu'elle n'était pas conditionnée par une consultation et disposait d'une grande «liberté de circulation»101. De même, on peut dire du phénomène «Bacis» que les efforts pour trouver une généalogie et une localisation exacte à ces figures signifient que l'on tombe dans le piège des créateurs anonymes de ces personnages.

99 Vid. une discussion sur cette question dans PARKE (qui propose Samos), op. cit. p. 64 sq.

100 Bien que ce soit seulement à titre d'hypothèse, on pourrait proposer les stades suivants :

a) La Sibylle comme figure littéraire, née sous l'influence des légendes et les nouvelles sur la mantique inspirée orientale. Ce personnage se trouverait dans des poèmes épiques du type des versions des Argonautes dont on suit les traces chez Eumélos.

b) Attribution d'oracles du type «Bacis», d'abord en rapport avec les territoires les plus proches de ses prétendues origines (cf., par exemple, la théorie des «Mermnades» de Parke).

c) Parution des centres "sibyllins".

101 Sans vouloir compliquer cette intervention avec une polémique sur "Mündlichkeit und Schriftlichkeit", mon opinion est que l'oracle en vers est l'un des types de poésie les mieux adaptés à une composition et à une diffusion écrite. Davantage encore les oracles "sibyllins" pseudépigraphes. 
Ces prophéties ont connu un grand succès dans les moments les plus délicats de la vie des Grecs, et leur extension pendant la période des guerres contre la Perse en est le témoignage. Pendant le IVe siècle, on en aperçoit une revitalisation, en rapport, d'un côté, avec des conflits entre plusieurs communautés (et une présence abondante, ainsi que d'autres oracles, dans l'historiographie locale) et, de l'autre côté, avec la résurgence de la menace perse et le pouvoir croissant des Macédoniens. On enregistre à cette époque un phénomène curieux. Des études de Peek $^{102}$ et Parke ${ }^{103}$ sur l'oracle épigraphique IG II $^{2} 4968$, daté ca 340 av. J.-C., ont contribué à résoudre l'énigme de cette inscription : il s'agirait d'un oracle né sous l'attribution de Bacis pendant la période des Guerres Médiques, mais réutilisé (et peut-être remanié) en 340 av. J.-C. pour mettre en garde contre deux dangers imminents: les Perses (à nouveau) et Philippe.

De la même façon que l'époque d'Alexandre avait provoqué la parution «en scène» de plusieurs centres sibyllins, un phénomène semblable va être enregistré avec les prédictions sibyllines, surtout après sa mort. Pendant la période hellénistique (et spécialement dans des cercles juifs) va commencer à se consolider une perspective apocalyptique qui connaîtra un développement extraordinaire dans le corpus des oracles sibyllins postérieurs. On verra naître une abondante série d'oracles qui annoncent la chute des rois hellénistiques. Mais il y a un motif, avec des racines assez profondes et dans la culture sémitique et (peut-être par influence ancienne) dans la culture grecque, qui va connaître un fort élan: la succession des Empires ${ }^{104}$. Il s'agit d'une théorie d'origine perse, ou plus concrètement mède (en dernier lieu il faut remonter à Zoroastre), qui, dans sa version la plus ancienne (dont les traces se trouvent déjà chez Hérodote et, plus tard, Ctésias de Cnide au IVe siècle), les Empires qui se succèdent sont l'Assyrie, la Médie et la Perse. À cette séquence, on ajoutera la Macédoine et, plus tard, Rome.

102 Attische Versinschriften, in Abhandlungen der sächsischen Akademie der Wissenschaften zu Leipzig, Phil.-hist. Klasse, Band 69, 2 (1980), p. 46-68 (n 53$)$.

103 What Kind of Oracle is $I G I I^{2}, 4968$ ?, in $Z P E, 60$ (1985), p. 93-96.

104 Cf. D. FLusser, The four empires in the Fourth Sibyl and in the Book of Daniel, in IOS, 2 (1972), p. 148-175. Sur le thème des rapports et des influences entre la Perse et la Grèce dans le domaine des prophéties pendant les périodes hellénistique et impériale, c'est le travail de J. BIDEZ -F. CUMONT, Les Mages hellénisés (Zoroastre, Ostanès et Hystaspe d'après la tradition grecque), 2 vol., Paris, 1938, qui reste toujours fondamental. 
L'auteur juif inconnu qui, au IIe siècle av. J.-C., s'est mis à composer les premiers vers de la collection des Oracles sibyllins procédait à un travail intégrateur (si on veut éviter ici le terme de "syncrétisme») entre Orient et Occident qui aura une répercussion extraordinaire pendant le reste de l'Antiquité et au Moyen Âge. La doctrine juive se revêtait d'une forme littéraire développée en Grèce (mais sans écarter des antécédents orientaux), combinée avec des structures et des thèmes qui n'étaient pas étrangers à la littérature religieuse juive et sous l'autorité d'une figure qui avait des précédents sémitiques, mais s'était enracinée très tôt dans le monde Grec : la mystérieuse Sibylle.

Departamento de Filología Griega

Emilio SuÁrez de LA TORRE

Facultad de Filosofia y Letras

Universidad de Valladolid

E - 47002 V ALLADOLID 